\title{
A Robust Multi-RAT VANET/LTE for Mixed Control \& Entertainment Traffic
}

\author{
Noha M. Sadek, Hassan H. Halawa, Ramez M. Daoud, Hassanein H. Amer \\ Electronics and Communications Engineering Department, American University in Cairo, Cairo, Egypt \\ Email: nohams@yahoo.com, hhhalawa@ieee.org, rdaoud@ieee.org, hamer@aucegypt.edu
}

Received 5 March 2015; accepted 8 April 2015; published 10 April 2015

Copyright @ 2015 by authors and Scientific Research Publishing Inc.

This work is licensed under the Creative Commons Attribution International License (CC BY).

http://creativecommons.org/licenses/by/4.0/

(c) (i) Open Access

\begin{abstract}
Intelligent Transportation Systems (ITS) have been receiving significant interest from various stakeholders worldwide. ITS promise major enhancements to the efficiency, safety, convenience and sustainability of transportation systems. To satisfy the diverse vehicular application requirements, this paper proposes an integration of IEEE 802.11-based VANET and LTE cellular network using mobile vehicular gateways. IEEE $802.11 \mathrm{~g}$ is used for V2V communications and LTE for V2I communications. A burst communication technique is applied to prevent packet losses in the critical uplink ITS traffic. A performance simulation-based study is conducted to validate the feasibility of the proposed system in an urban vehicular environment. The system performance is evaluated in terms of data loss, data rate, delay and jitter. The results indicate that the proposed Multi-RAT system offers acceptable performance that meets the requirements of the different vehicular applications.
\end{abstract}

\section{Keywords}

Heterogeneous Networks, Intelligent Transportation Systems, Vehicular Networks, VANET, LTE, Wi-Fi

\section{Introduction}

Intelligent Transportation Systems (ITS) have been receiving significant interest from various stakeholders worldwide. Automotive companies, governmental entities, standardization organizations and the research community are all focusing on the design and deployment of ITS. ITS promise major enhancements to the efficiency, safety, convenience and sustainability of transportation systems. Specifically, ITS aim at improving road safety, alleviating urban traffic congestion and offering ubiquitous Internet access for passengers [1].

Vehicular networks are believed to play a crucial role in future ITS due to the wide variety of services that 
they can offer. Vehicular network applications can be broadly classified into 3 main categories, namely active road safety, traffic efficiency and management, and infotainment applications [2]. Safety applications are the first type of applications that aim at enhancing passengers' safety on the roads by notifying vehicles about dangerous incidents in their vicinity. This can be accomplished by exchanging information like vehicle speed, position and distance heading between vehicles and the infrastructure, which is then used to predict and avoid risky situations [3].

Besides safety applications, vehicular networks also support traffic efficiency and management applications. The primary objective of applications in this category is to improve traffic flow and enhance traffic coordination and management. Drivers can avoid congestion and find the most efficient route to their destinations with minimum delay. Additionally, vehicular traffic on the streets will be balanced which will lead to efficient use of the capacity of streets and junctions. This will in turn result in energy savings, pollution reduction and a decrease in travel time. The third type of vehicular applications is infotainment applications, also called comfort or entertainment applications. This class of applications provides information that delivers global Internet services and enhances the passengers' comfort, convenience and entertainment.

In summary, vehicular applications enable the gathering and dissemination of information among vehicles (V2V), and between transportation infrastructure and vehicles (V2I) with the goal of assisting passengers to travel safely, efficiently and conveniently. Reliability, mobility support and low-latency are thus critical to satisfy the performance requirements of the different vehicular applications. On one side of the spectrum, infotainment applications have high-bandwidth demands and QoS-sensitive requirements [4]. While, on the other side of the spectrum, safety-critical applications are characterized by low latency and high message delivery rate. To support safety application demands, a large amount of data traffic needs to be exchanged between vehicles and Base Stations (BSs). This will consequently add a heavy burden to the BSs, which is not likely to be accepted by network operators. Additionally, these extra traffic connections increase the effect of interference and thus increase data error rate. Moreover, this also causes an increase in packet delays due to resource depletion. Furthermore, the scheduler at the BS may have difficulties scheduling transmissions within the tight delay bounds required for safety-critical applications [5].

To cater to the diverse vehicular application requirements, this paper proposes an integration of IEEE 802.11based VANET and LTE cellular network using mobile vehicular gateways. The proposed heterogeneous vehicular network combines two technologies with long-range and short-range coverage, namely LTE and Wi-Fi (IEEE 802.11) respectively. Each technology has a different objective and their integrated deployment will improve the vehicular system performance. On one hand, Wi-Fi offers a relatively high capacity at a very low cost and it has a high market penetration. However, it has a low coverage range compared to LTE. This makes it suitable for use as an access network inside the vehicle and for $\mathrm{V} 2 \mathrm{~V}$ communication between nearby vehicles. On the other hand, LTE offers a wide coverage and better Quality of Service (QoS) but, it requires costly licensed spectrum and is lacking behind Wi-Fi in terms of economies of scale [6]. These characteristics fit with the long range communication requirements of the V2I backhaul network. By integrating Wi-Fi with LTE, high capacity is coupled with long-range communication to improve the overall performance of the vehicular system. IEEE $802.11 \mathrm{~g}$ (Wi-Fi) has been selected because of its popularity and low cost. It offers users a uniform and mass-standard connectivity as the same standard is widely used in various parts of cities like hot-spots, tourist centers and information points [7].

In the proposed heterogeneous network, there are mainly two types of vehicles: Gateway Vehicles (GVs) and Ordinary Vehicles (OVs). GVs are equipped with both LTE and Wi-Fi interfaces while OVs are only Wi-Fi enabled. A GV can be connected to two networks simultaneously: the LTE network using its E-UTRAN interface and to other OVs through its Wi-Fi interface. The GV can thus serve as a mobile gateway (i.e., relay node) for other OVs in its vicinity to access the LTE network. This can be accomplished by receiving data from OVs (using the Wi-Fi interface) and relaying it to the LTE network (via the LTE interface). OPNET Modeler [8] is used for the design, implementation and evaluation of the proposed network model.

The rest of the paper is organized as follows. Section 2 provides a background about vehicular networking research. Section 3 describes the proposed architecture. Section 4 presents the results and performance evaluation of the proposed architecture. Finally, the paper is concluded in Section 5.

\section{Background}

This section presents some studies that evaluated the feasibility of using IEEE 802.11 and cellular networks to 
support vehicular networking applications.

In [9], an attempt was made to solve the traffic control problem in an urban environment using Wi-Fi where a burst communication technique was used to eliminate packet losses. The authors in [10] proposed a heterogeneous LTE/Wi-Fi vehicular system that supports both infotainment and ITS traffic control data. Other studies ([1] [5] [11]-[14]) evaluated the performance of heterogeneous LTE-VANET (Vehicle Ad-hoc Networks) networks, where LTE was the backhaul network and VANET was used for inter-vehicle communication. In [5], LTE was used to exchange Cooperative Awareness Messages (CAMs) between clusters while, Wi-Fi delivered in-cluster information. Reference [11] envisioned a heterogeneous LTE/IEEE 802.11p network that provides multimedia communication services over spatially apart vehicular groups and a cluster head election mechanism was proposed. A cooperative protocol based on coalition game theory was introduced in [12] to disseminate data in LTE/VANET network. Reference [13] investigated the delivery of real-time streaming of scalable video coded (SVC) video over vehicle-to-infrastructure (V2I) links. The inter-vehicles to infrastructure (V2V2I) model considered in [1] again used IEEE 802.11p for V2V communications and LTE for V2I communications. In [14], the LTE network was used as a cluster management infrastructure for the IEEE 802.11p VANET. The performance was compared with the decentralized VANET architecture for an urban sensing application. Many of the studies in the field of vehicular communications only investigate a single type of application and either cover V2I or V2V communication only. The simultaneous support of safety, traffic and infotainment applications using both V2I and V2V communication has not been addressed in the literature. Accordingly, this perspective will be investigated in this study.

\section{Proposed Model}

\subsection{Network Architecture}

The envisioned WiFi-based VANET-LTE heterogeneous network architecture is shown in Figure 1. Vehicles that are equipped with both LTE and Wi-Fi interfaces are referred to as Gateway Vehicles (GVs) whereas, only Wi-Fi is supported on-board Ordinary Vehicles (OVs). A GV is under the coverage region of at least one LTE eNodeB, and its LTE and Wi-Fi interfaces are both activated. On the other hand, an OV either lacks an LTE interface or is not present in an LTE coverage area. In other words, it is assumed that the LTE interface is either absent or disabled on OVs.

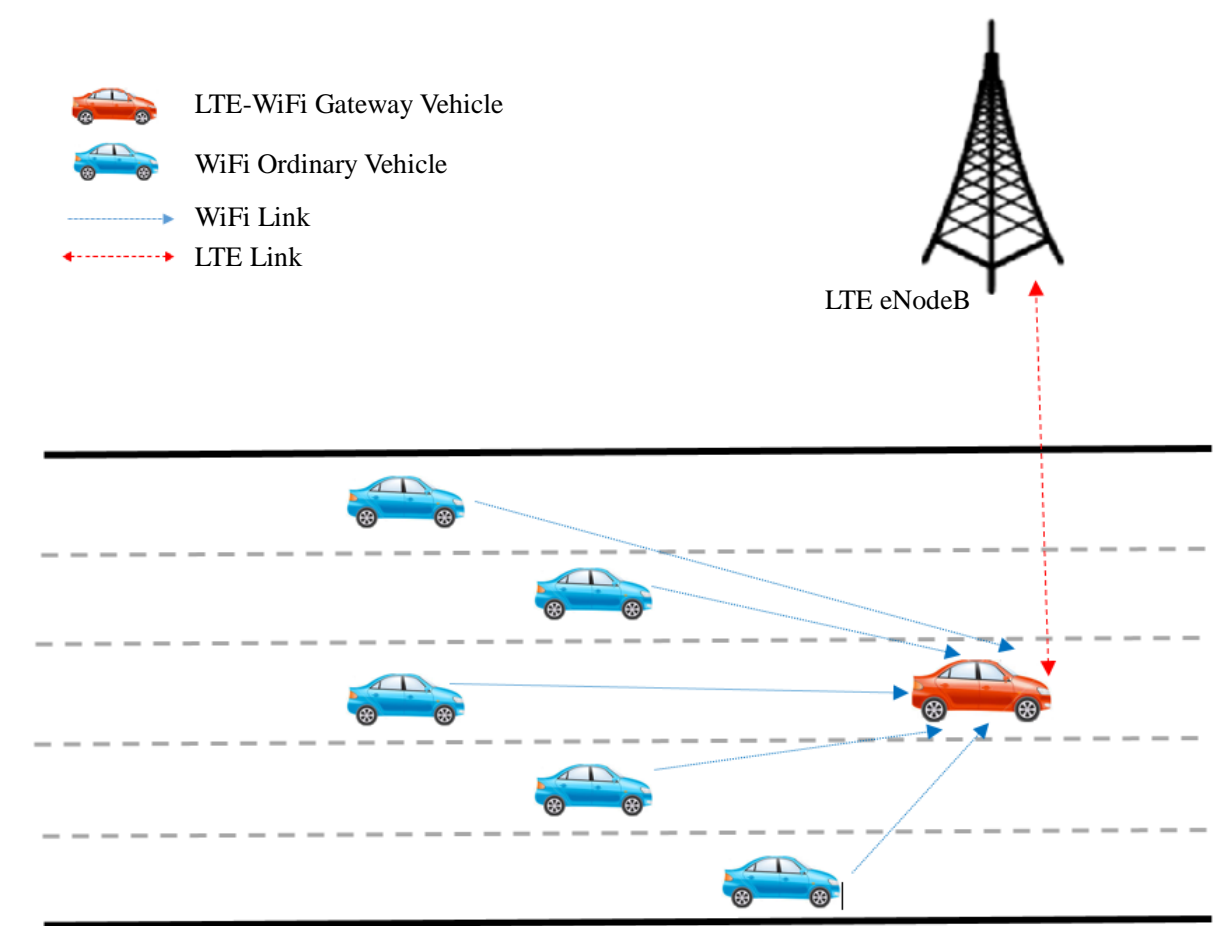

Figure 1. VANET-LTE network architecture. 
This paper builds on the model introduced in [10] which consists of seven cells arranged in a hexagonal honey-cell layout where each cell is covered by one eNodeB. The vehicles are modeled moving in a radial path between the 7 cells in an urban traffic model where vehicles move at the maximum speed of $60 \mathrm{Km} / \mathrm{hr}$. The proposed architecture divides the vehicular network into Vehicle-to-Vehicle (V2V), Vehicle-to-Infrastructure (V2I), On-board Vehicle Communication (OVC), and backhaul connection. The V2V network allows communication between GVs and OVs through Wi-Fi. The V2I network between the vehicle and LTE eNodeB provides access to the LTE core network. The OVC network consists of a Wi-Fi Access Point (AP), passengers' devices and a vehicular OnBoard Unit (OBU) for ITS data. In this scenario, LTE is the backhaul link used to access the Internet and the connectivity is shared to vehicular users using Wi-Fi as the last mile link. The LTE backhaul links are full duplex. Typically, an LTE eNodeB is deployed alongside the road and the vehicles are under the coverage of the different eNodeBs. More details about the system architecture can be found in [10].

From the data flow perspective, GVs sample and gather the information from OVs (through Wi-Fi) then, in turn send periodically the relevant data to the infrastructure (via LTE). Data is exchanged between the GVs and LTE eNodeBs in both the downlink and uplink directions. On the downlink, LTE eNodeBs unicast the data to the GVs where both ITS and infotainment traffic are sent over the LTE link yet, they are routed differently inside the vehicle based on the intended destination. ITS data is sent to the vehicle's OBU via Ethernet for further processing and decision making. On the other hand, infotainment traffic is sent to the passengers' devices through Wi-Fi. On the uplink, GVs forward the traffic data (collected from OVs) to the LTE infrastructure at a pre-determined transmission rate.

In order to reduce the amount of traffic exchanged between vehicles and eNodeBs, a clustering strategy is employed. The GV is the cluster head which maintains the status of the cluster. Only the LTE-enabled cluster head is allowed to receive/transmit data from/to eNodeBs through LTE interfaces. Every OV transmits small data packets called Cooperative Awareness Messages (CAMs) to their corresponding cluster head/GV, providing state information such as speed or location. Such CAMs are transmitted every $100 \mathrm{~ms}$ [5]. It is considered that the vehicle clusters are already formed and that in each cluster, vehicles are moving together across all seven cells along the trajectory described in [10]. Thus, cluster members can be assumed not to vary throughout the journey. A single cluster of vehicles consists of $1 \mathrm{GV}$ and 5 OVs.

\subsection{Simulation Parameters}

The network model is simulated using OPNET Modeler [8] network simulator. The various network configuration parameters are shown in Table 1. eNodeB, GV LTE and downlink ITS parameters are all obtained from the model explained in [10]. CAMs are transmitted from OVs to GV where CAM size is 40 Bytes and transmission interval is $100 \mathrm{~ms}$ [5]. GV then aggregates CAMs' data (12000 Bytes) and sends it on the LTE uplink channel every 30 seconds. 33 simulation runs were performed with different random seeds in order to ensure statistical accuracy. It is important to note that all results presented in this paper are subjected to a $95 \%$ confidence analysis.

\subsection{Performance Evaluation Metrics}

This section describes the performance evaluation metrics of the proposed model. The network performance is evaluated in terms of data rate, Data Loss Ratio (DLR), delay and jitter parameters, defined as follows:

- Data Rate (in Bytes/sec) is defined as the sum of the data bytes received at the destination averaged over time.

- Data Loss Ratio (DLR) is defined as the ratio between dropped packets that do not reach the destination and the total number of packets sent from the source to the destination.

- Delay (in seconds) specifies the time elapsed between sending the request from the source and the reception of the response at the source. This metric serves as a measure of the average overall delay of the packets for a particular node.

- Jitter (in seconds) is defined as the packet delay variation. This metric is calculated as the standard deviation of packet delay for all packets sent over the network for a particular node.

\section{Simulation Results}

In this paper, the overall system performance, as specified by the communication requirements imposed by 
Table 1. Simulation parameters.

\begin{tabular}{|c|c|}
\hline Parameter & Value \\
\hline \multicolumn{2}{|l|}{ eNodeB } \\
\hline Transmit Power & 10 Watts \\
\hline Antenna Gain & $18 \mathrm{dBi}$ \\
\hline MIMO & $2 \times 2$ \\
\hline Bandwidth & $10 \mathrm{MHz}$ \\
\hline Frequency Band & $1.8 \mathrm{GHz}$ \\
\hline Rx Sensitivity & $-123 \mathrm{dBi}$ \\
\hline Duplexing Technique & FDD \\
\hline Cell Radius & $1.5 \mathrm{Km}$ \\
\hline ISD & $2.6 \mathrm{Km}$ \\
\hline Transmit Power & 10 Watts \\
\hline \multicolumn{2}{|l|}{ GV } \\
\hline Transmit Power & 0.2 Watts \\
\hline Antenna Gain & $0 \mathrm{dBi}$ \\
\hline MIMO & $1 \times 2$ \\
\hline Rx Sensitivity & $-106 \mathrm{dBi}$ \\
\hline Shadow Fading Standard Deviation & $4 \mathrm{~dB}$ \\
\hline Downlink ITS IPT & $120 \mathrm{~s}$ \\
\hline Downlink ITS Size & 1024 Bytes \\
\hline Uplink ITS IPT & $30 \mathrm{~s}$ \\
\hline Uplink ITS Size & 12000 Bytes \\
\hline \multicolumn{2}{|l|}{$\mathrm{OV}$} \\
\hline CAM Transmission Interval & $100 \mathrm{~ms}$ \\
\hline CAM Size & 40 Bytes \\
\hline
\end{tabular}

different types of vehicular networking applications, is investigated. For this purpose, the foremost emphasis is on evaluating the data loss, data rate, delay and jitter of vehicular V2V and V2I applications in a realistic urban simulation environment. For all presented scenarios, the aforementioned performance evaluation metrics (data rate, DLR, delay and jitter) were analyzed. A 95\% confidence analysis was performed for all presented results.

\subsection{No Burst Model}

In a video streaming service environment, it is important to maintain the DLR threshold below 1\% [15] [16] such that the QoS requirement of video streaming service users is satisfied. Additionally, the performance of video streaming depends greatly on delay and jitter. According to [17], the maximum acceptable video packet delay is set to $150 \mathrm{~ms}$ and the maximum allowable jitter is $50 \mathrm{~ms}$. For traffic control data, since most of the applications are time-critical, the end-to-end delay must be between 100 and 500 ms [18].

Table 2 shows the results obtained for vehicular video, downlink and uplink ITS traffic. It can be seen that the maximum video streaming traffic delay is $12.94 \mathrm{~ms}$, while the maximum jitter is 5.84 ms. For downlink ITS traffic, a maximum delay of $22.27 \mathrm{~ms}$ and jitter of $6.7 \mathrm{~ms}$ is observed. As for uplink ITS traffic, the maximum delay is $23.82 \mathrm{~ms}$ and the maximum jitter is $9.69 \mathrm{~ms}$. The obtained values are all below the above-mentioned benchmarks for ITS applications. It can be noted that the DLR for uplink traffic is $1.73 \%$. 
Figure 2 shows two packet losses in the uplink data traffic; one of them happens when the GV is in LTE cell 1 while the other one is in LTE cell 2. Due to the criticality of the safety-related information communicated in the uplink direction, a zero DLR is desired. So, in the next section, a burst recovery technique will be proposed to mitigate data losses in uplink ITS traffic.

The Wi-Fi V2V traffic results are presented in Table 3. Worst case values are considered where the DLR, delay and jitter values represent the upper bound of the resulting confidence interval. No data drops were reported for any of the OVs. In other words, all V2V data was successfully received by the GV via Wi-Fi. Additionally, the obtained delay values are far below the 100 ms constraint of the CAM V2V communication.

\subsection{Burst Recovery Mechanism}

Burst is a communication technique used to reduce or prevent data losses in wireless communication systems. This is accomplished by sending successive identical packets within a certain time frame and separated by a pre-defined period of time. In essence, if one of the original packets is lost, the other redundant packets will still carry the same information to the desired destination.

There are basically 3 parameters that characterize burst communication, namely $T_{\text {packet }}, T_{\mathrm{F} 2 \mathrm{~L}}$ and $T_{\text {burst }}$ [9]. $T_{\text {packet }}$ is the time between two successive packets within the same burst. $T_{\mathrm{F} 2 \mathrm{~L}}$ is the time between the first and the last packet in the same burst. Finally, $T_{\text {burst }}$ is the time between the first packets of two successive bursts.

$T_{\text {packet }}$ depends on the Inter-Packet Transmission Time (IPT) of the uplink traffic and on number of packets used in one burst, as follows:

Table 2. Confidence results of video, downlink, and uplink ITS traffic—No burst model.

\begin{tabular}{cccc}
\hline Metric & Video & Downlink ITS & Uplink ITS \\
\hline Data Rate (Bytes/sec) & {$[124311.1 ; 124338.5]$} & {$[8.46 ; 8.56]$} & {$[393.07 ; 397.69]$} \\
DLR (\%) & $0.55 \%$ & $0.8 \%$ & $1.73 \%$ \\
Delay (ms) & {$[12.91 ; 12.94]$} & {$[20.97 ; 22.27]$} & {$[22.79 ; 23.82]$} \\
Jitter (ms) & {$[5.59 ; 5.84]$} & {$[4.48 ; 6.7]$} & {$[7.47 ; 9.69]$} \\
\hline
\end{tabular}

Table 3. Results of V2V traffic-No burst model.

\begin{tabular}{cccccc}
\hline Metric & V2V OV1 & V2V OV2 & V2V OV3 & V2V OV4 & V2V OV5 \\
\hline Data Rate (Bytes/sec) & 400 & 400 & 400 & 400 & $0 \%$ \\
DLR (\%) & $0 \%$ & $0 \%$ & $0 \%$ & 1.63815 \\
Delay (ms) & 0.790889 & 1.11316 & 1.407288 & 0.4702 \\
Jitter (ms) & 0.443402 & 0.41698 & 0.387431 & 0.35542 \\
\hline
\end{tabular}

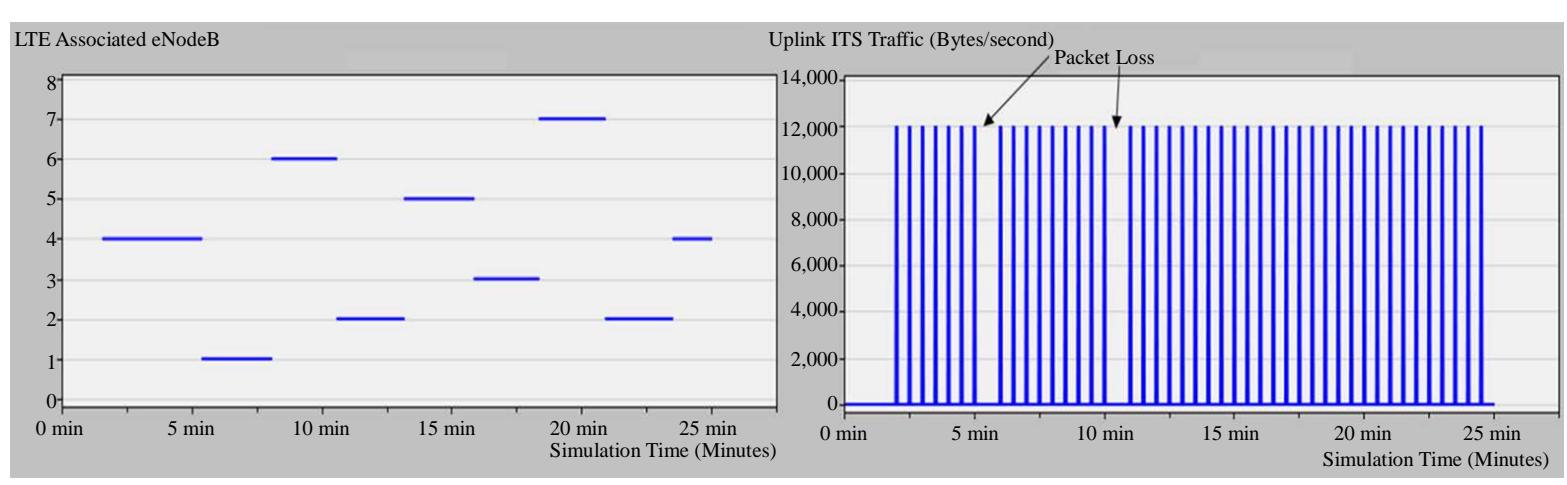

Figure 2. Uplink ITS traffic and LTE associated eNodeB. 


$$
T_{\text {packet }} \leq \frac{I P T}{N_{\text {burst }}}
$$

where IPT is the Inter-Packet Transmission Time of the uplink traffic and $N_{\text {burst }}$ is the number of packets per burst. $T_{F 2 L}$ depends on the number of packets used in one burst and must satisfy the following constraint:

$$
T_{F 2 L}=T_{\text {packet }} \times\left(N_{\text {burst }}-1\right)
$$

$T_{\text {burst }}$ must be less than or equal the IPT, which corresponds to the time needed for traffic and routing updates.

$$
T_{\text {burst }} \leq I P T
$$

The minimum number of packets that can be used in one burst is two. A two-packet burst is studied to optimize the LTE channel utilization and minimize the network load. From Equation (1) and for a 30-sec uplink IPT, Tpacket must be smaller than or equal to $15 \mathrm{sec}$. In case of 2 packets per burst, $T_{F 2 L}=T_{\text {packet }}$ from Equation (2).

Figure 3 shows a typical burst communication for ITS uplink traffic. It is clear that two packets were lost from the original uplink traffic, however their burst replicas arrived successfully which indicates that no actual uplink ITS data was lost. Table 4 shows the results obtained for vehicular video, downlink and uplink ITS traffic for the Burst model. Table 5 shows Wi-Fi V2V traffic results for the Burst model. All received data, delay and jitter are within the acceptable limits of ITS applications.

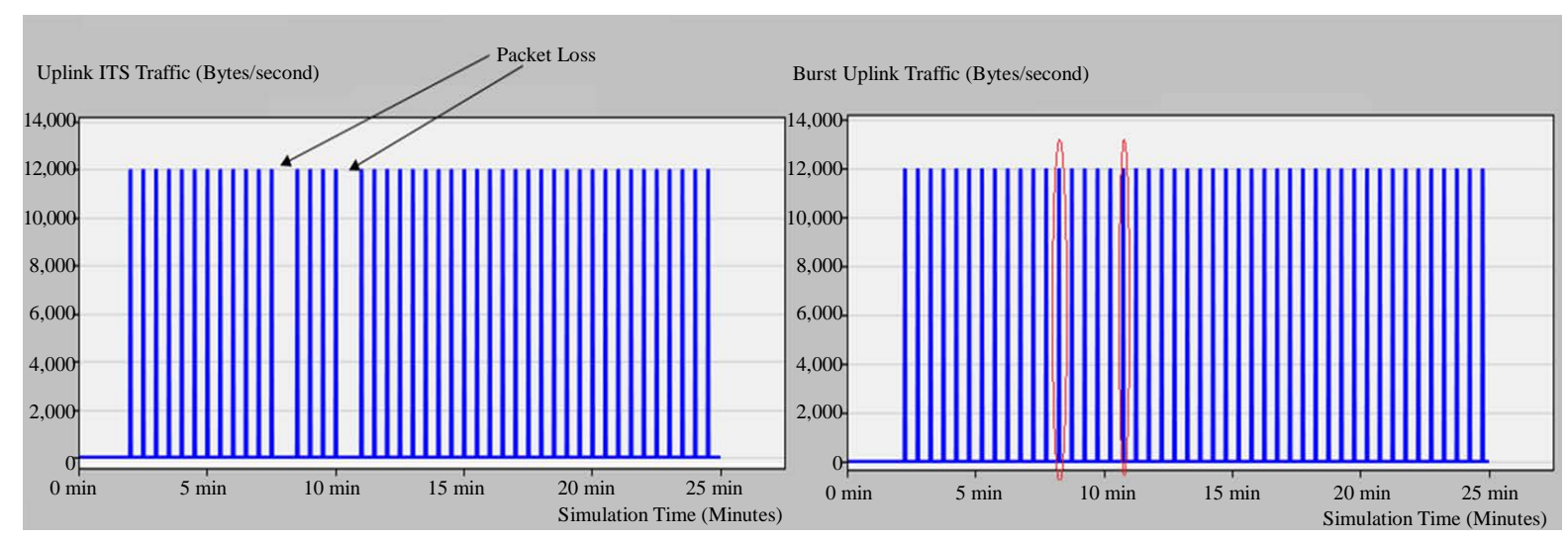

Figure 3. Uplink ITS traffic with Burst.

Table 4. Confidence results of video, downlink, and uplink ITS traffic—Burst model.

\begin{tabular}{cccc}
\hline Metric & Video & Downlink ITS & Uplink ITS \\
\hline Data Rate (Bytes/sec) & {$[124321.4 ; 124347.2]$} & {$[8.39 ; 8.54]$} & {$[393.08 ; 397.1]$} \\
DLR (\%) & $0.5 \%$ & $1.8 \%$ & $0 \%$ \\
Delay (ms) & {$[12.9 ; 12.93]$} & {$[21.52 ; 22.53]$} & {$[23.4 ; 24.36]$} \\
Jitter (ms) & {$[5.56 ; 5.66]$} & {$[5.32 ; 7.58]$} & {$[8.69 ; 11.13]$} \\
\hline
\end{tabular}

Table 5. Results of V2V traffic-Burst model.

\begin{tabular}{cccccc}
\hline Metric & V2V OV1 & V2V OV2 & V2V OV3 & V2V OV4 & 400 \\
\hline Data Rate (Bytes/sec) & 400 & 400 & 400 & $0 \%$ & $0 \%$ \\
DLR (\%) & $0 \%$ & $0 \%$ & 1.406 & 0.47 \\
Delay (ms) & 0.79 & 1.113 & 0.3867 & 0.3547 \\
Jitter (ms) & 0.4436 & 0.4178 & 0.4119 \\
\hline
\end{tabular}


To conclude, the delay, jitter and data drops for both uplink and downlink ITS traffic, and video traffic are within the acceptable limits using the burst technique with only two packets per burst. The obtained simulation results thus show that the proposed system simultaneously satisfies vehicular and infotainment application requirements.

\section{Conclusions}

It is anticipated that ITS will play a vital future role in improving traffic efficiency, safety, comfort and emissions. Such systems rely on advanced mobile and wireless communication systems to satisfy the different vehicular applications' requirements and to accommodate the increasing vehicles' fleet. In this paper, an integrated IEEE802.11g-based VANET and LTE heterogeneous vehicular network was proposed where infotainment traffic was sent in addition to ITS control traffic in an urban vehicular environment. The system performance was evaluated in terms of data loss ratio, data rate, delay and jitter.

Data losses in uplink ITS data traffic were initially observed so, a Burst technique was proposed to prevent packet losses. A quantitative analysis was performed to determine the number of packets per burst, the interpacket and inter-burst intervals. It was found that a substantial improvement was achieved using a two-packet Burst, where no packets were lost in the uplink direction. Additionally, for the given simulation scenario and network traffic load, it was shown that the proposed system meets both the video and ITS traffic application requirements. Thus, the feasibility of the proposed VANET-LTE heterogeneous system in urban vehicular environments was verified. Future work will focus on investigating more applications in the area of ITS.

\section{References}

[1] Imadali, S., Kaiser, A., Decremps, S., Petrescu, A. and Vèque, V. (2013) V2V2I: Extended Inter-Vehicles to Infrastructure Communication Paradigm. IEEE 5th Global Information Infrastructure Symposium, Trento, Italy, October 2013.

[2] Hossain, E., et al. (2010) Vehicular Telematics over Heterogeneous Wireless Networks: A Survey. Computer Communications, 33, 775-793. http://dx.doi.org/10.1016/j.comcom.2009.12.010

[3] Karagiannis, G., et al. (2011) Vehicular Networking: A Survey and Tutorial on Requirements, Architectures, Challenges, Standards and Solutions. IEEE Communications Surveys \& Tutorials, 13, 584-616. http://dx.doi.org/10.1109/SURV.2011.061411.00019

[4] Araniti, G., Campolo, C., Condoluci, M., Iera, A. and Molinaro, A. (2013) LTE for Vehicular Networking: A Survey. IEEE Communications Magazine, 51, 148-157. http://dx.doi.org/10.1109/MCOM.2013.6515060

[5] Tung, L., Mena, J., Gerla, M. and Sommer, C. (2013) A Cluster Based Architecture for Intersection Collision Avoidance Using Heterogeneous Networks. 12th IFIP/IEEE Annual Mediterranean Ad Hoc Networking Workshop (MedHoc-Net), Cesson-Sevigne, France, June 2013.

[6] Ghaleb, A., et al. (2013) Preservation of QOS across Hybrid LTE-WLAN Router. IET International Conference on Information and Communications Technologies (IETICT), Beijing, April 2013.

[7] Barbu, G. (2010) E-TRAIN-Broadband Communication with Moving Trains, Technical Report—Technology State of the Art. International Union of Railways, Paris.

[8] Official Site of OPNET: $\underline{w w w . o p n e t . c o m}$

[9] Daoud, R., EI-Dakroury, M., Elsayed, H., Amer, H. and EISoudani, M. (2007) WiFi Architecture for Traffic Control Using MIPv6. IEEE Mediterranean Conference on Control and Automation MED, Athens, 27-29 July 2007, 1-5. http://dx.doi.org/10.1109/MED.2007.4433921

[10] Sadek, N., Halawa, H., Daoud, R., Amer, H. and Ali, N. (2015) Heterogeneous LTE/Wi-Fi Architecture for ITS Traffic Control and Infotainment. International Conference on Electrical Systems for Aircraft, Railway, Ship Propulsion and Road Vehicles, Aachen, March 2015.

[11] Sivaraj, R., Gopalakrishna, A., Chandra, M. and Balamuralidhar, P. (2011) QoS-Enabled Group Communication in Integrated VANET-LTE Heterogeneous Wireless Networks. IEEE 7th International Conference on Wireless and Mobile Computing, Networking and Communications (WiMob), October 2011.

[12] Li, Y., Ying, K., Cheng, P., Yu, H. and Luo, H. (2012) Cooperative Data Dissemination in Cellular-VANET Heterogeneous Wireless Networks. 4th International High Speed Intelligent Communication Forum (HSIC), Nanjing, May 2012.

[13] Yaacoub, E., Filali, F. and Abu-Dayya, A. (2013) SVC Video Streaming over Cooperative LTE/802.11p Vehicle-to- 
Infrastructure Communications. IEEE World Congress on Computer and Information Technology (WCCIT), Tunis, June 2013.

[14] Remy, G., Senouci, S., Jan, F. and Gourhant, Y. (2011) LTE4V2X: LTE for a Centralized VANET Organization. IEEE Global Telecommunications Conference (GLOBECOM), Houston, 5-9 December 2011, 1-6. http://dx.doi.org/10.1109/GLOCOM.2011.6133884

[15] Islam, M. and Chowdhury, M. (2013) Study of Inter-Cell Interference and Its Impact on the Quality of Video Conference Traffic in LTE Network. M.Sc. Thesis, Blekinge Institute of Technology, Sweden.

[16] Ramli, H., Basukala, R., Sandrasegaran, K. and Patachaianand, R. (2009) Performance of Well Known Packet Scheduling Algorithms in The Downlink 3GPP LTE system. IEEE 9th Malaysia International Conference on Communications (MICC), Kuala Lumpur, 15-17 December 2009, 815-820. http://dx.doi.org/10.1109/MICC.2009.5431383

[17] International Telecommunication Union (ITU) (2008) Subjective Video Quality Assessment Methods for Multimedia Applications. Recommendation ITU-T P.910, Geneva.

[18] Mir, Z. and Filali, F. (2014) LTE and IEEE 802.11p for Vehicular Networking: A Performance Evaluation. EURASIP Journal on Wireless Communications and Networking, 2014, 89. http://dx.doi.org/10.1186/1687-1499-2014-89 